\title{
PRESENCIA NO DESEADA DEL SPLASH OVER EN EQUIPOS QUE UTILIZAN SENSORES INDUCTIVOS PARA EL MONITOREO DE LA VELOCIDAD CONTROLADA EN VEHÍCULOS AUTOMOTORES
}

\author{
Silvio MonTEIRO ${ }^{1}$ \\ MAURÍCIO RÉCHE ${ }^{2}$ \\ Altair S. DE Assis ${ }^{3}$
}

\section{RESUMEN}

Este artículo busca evaluar los instrumentos que supervisan el flujo de tráfico electrónicamente mediante el uso sensores de superficie inductiva con base en la modificación del campo magnético. Más específicamente, estudiamos la posibilidad de detecciones falsas de velocidad debido a la manifestación del fenómeno llamado splash over - que es un espacio registrado en las líneas del campo magnético por fuera del área de medición. Demostramos a través de la literatura y de simulaciones prácticas, el daño que puede ser causado por el splash en la medición de la velocidad correcta y la identificación del vehículo infractor. También se presentan soluciones para inhibir las mediciones de velocidad no deseadas debido a este efecto.

PALABRAS CLAVE: Supervisión del tráfico, medición de velocidad, metrología legal, splash over, campo magnético.

\section{UNDESIRED SPLASH OVER ON EQUIPMENTS USING INDUCTIVE SENSORS FOR MONITORING CONTROLLED SPEED IN AUTOMOTIVE VEHICLES}

\section{ABSTRACT}

This article aims to evaluate instruments that oversight traffic flow electronically, using inductive surface sensors, based on the change of local magnetic field. More specifically, we study the possibility of false speed detections, due to the manifestation of the phenomenon called splash over — which means a space splash of the magnetic field lines out of

1 Ingeniero Mecánico, Río de Janeiro Universidad Estatal; Master en Metrología y Calidad, Instituto Nacional de Metrología, Calidad y Tecnología. Tecnólogo investigador del Instituto Nacional de Metrología, Calidad y Tecnología (INMETRO). Río de Janeiro, Brasil.

2 Físico, Río de Janeiro Universidad Federal; Master de Sistemas Integrados de Gestión de la Universidad Federal Fluminense. Tecnólogo investigador del Instituto Nacional de Metrología, Calidad y Tecnología (INMETRO). Río de Janeiro, Brasil.

3 Físico de la Universidad Federal Fluminense, Río de Janeiro; Master en Física de Plasma de la Universidad Federal Fluminense; Doctor en Filosofía. Física del plasma de la Universidad Estatal de Campinas; Profesor Titular, Departamento de Matemática Aplicada de la Universidad Federal Fluminense. Niterói, Brasil.

Autor de correspondencia: de Assis A. (Altair): Caixa Postal 100294. Post code: 24001-970, Niterói, Rio de Janeiro Brazil. Correo electrónico: altair@vm.uff.br
Historia del artículo:

Artículo recibido: 10-IV-2013/ Aprobado: 18-II-2017

Disponible online: 30 de agosto de 2017

Discusión abierta hasta octubre de 2018 
the measuring zone. We show, through the literature, and practical simulations, the damage that can be caused by splash on the correct speed measurement and on the identification of the speeding vehicle. The article also provides solutions to inhibiting unwanted velocity measurements due to this effect.

KEY WORDS: Oversight Traffic, Speed Meters, Legal Metrology, Splash Over, Magnetic Field.

\section{PRESENÇA NÃO DESEJADA DO SPLASH OVER EM INSTRUMENTOS DE SENSORES INDUTIVOS QUE CONTROLAM OS VEÍCULOS AUTOMOTORES}

\section{RESUMO}

Este artigo trata de instrumentos que supervisionam o tráfico eletronicamente mediante o uso de sensores de superfície inductive, baseado na modificação do campo magnético. Mais especificamente, a possibilidade de detecções falsas de veículos devido à manifestação do fenômeno chamado splash over. Demonstrando através dos fundamentos bibliográfico e simulações práticas, o dano que pode ser causado pelo splash over sobre a medida da velocidade correta e a identificação do mensurando. Também é possível expor soluções para a inibição de falhas devido a este fenômeno.

PALAVRAS CHAVE: Supervisão do trânsito, medidores de velocidade, metrologia legal, splash over, campo magnético.

\section{INTRODUCCIÓN}

Los accidentes de tránsito según la Organización Mundial de la Salud (2004) registran más de 1.2 millones de fatalidades por año en todo el mundo y lesionan a cerca de otros 50 millones.

El exceso de velocidad por sí solo, o combinado con otros factores, pueden ser considerados como un agente fundamental en los accidentes de tránsito. Los datos de la Policía Federal de Carreteras de Brasil sobre los accidentes ocurridos en las vías federales de este país confirman el impacto del exceso de velocidad en las estadísticas de accidentes de tráfico, directamente o debido a otros factores (Rizzoto, 2010).

Controlar la velocidad electrónicamente a través de instrumentos de medición de la velocidad de los vehículos a motor se considera un poderoso aliado en el esfuerzo para frenar el exceso de velocidad y los accidentes resultantes. Tales herramientas han sido ampliamente utilizadas en los últimos 30 años en diversas partes del mundo.

Los principales medios utilizados comercialmente para la medición de la velocidad vehicular son:

a) Radar: Su funcionamiento se basa en el movimiento de los vehículos. El instrumento emite una onda electromagnética, por lo general ondas de radiofrecuencia, que alcanzan un objeto, éstas se reflejan y son capturadas por el radar receptor. El movimiento del objetivo dará lugar a un cambio en la frecuencia emitida. La diferencia entre las frecuencias emitidas y reflejadas es traducida por el decodificador en el radar como un valor de velocidad.

b) Ópticos (láser): Los instrumentos con esta tecnología para medir las velocidades utilizan ondas monocromáticas emitidas por un láser. El tiempo que le toma a un pulso láser para alcanzar el objetivo y volver es directamente proporcional a la 
distancia de dicho objetivo. Por lo tanto la emisión de varios pulsos en el tiempo pueden determinar la distancia a un vehículo en este intervalo de tiempo y por lo tanto la velocidad.

c) Procesamiento de imágenes: La medición de la velocidad utilizando imágenes se realiza a través de una estructura equipada con cámaras digitales de alta capacidad. Su principio de funcionamiento se basa en el uso de algoritmos de procesamiento de imágenes. Los puntos de medi- ción son prácticamente seguros en su propia imagen y se manejan a través de software. La combinación de múltiples puntos de detección hace posible medir la velocidad.

d) Los sensores inductivos: La detección inductiva se discute más en detalle en la Sección 2.

Cada uno de estos métodos tienen limitaciones inherentes a la tecnología utilizada lo que puede provocar fallos en la detección del objeto medido, como se muestra en la Tabla 1.

TABLA 1. VENTAJAS Y DESVENTAJAS DE LAS PRINCIPALES TECNOLOGÍAS UTILIZADAS EN LA DETECCIÓN DE LOS VEHÍCULOS A MOTOR

\begin{tabular}{|c|c|c|}
\hline Tecnología & Ventajas & Desventajas \\
\hline $\begin{array}{l}\text { Sensores } \\
\text { Inductivos }\end{array}$ & $\begin{array}{l}\text { - Diseño flexible para satisfacer diversas } \\
\text { aplicaciones; } \\
\text { - Tecnología bien entendida; } \\
\text { - Proporciona parámetros básicos del tráfico } \\
\text { (volumen, presencia, ocupación, velocidad); } \\
\text { - No se ve afectado por las variaciones } \\
\text { climáticas; } \\
\text { - Puede proporcionar datos sobre } \\
\text { clasificación y tipos de vehículos; } \\
\text { - Bajo costo de instalación y mantenimiento }\end{array}$ & $\begin{array}{l}\text { - La instalación requiere un corte en el pavimento; } \\
\text { - Una instalación inadecuada disminuye vida útil del } \\
\text { pavimento; } \\
\text { - Instalación y mantenimiento implican el cierre de la vía; } \\
\text { - Los sensores instalados en la vía están sujetos al } \\
\text { desgaste; } \\
\text { - La precisión en la detección puede disminuir cuando el } \\
\text { diseño requiere la detección de una gran variedad de } \\
\text { clases de vehículos; } \\
\text { - Un ajuste incorrecto puede provocar splash over. }\end{array}$ \\
\hline Radar (Doppler) & $\begin{array}{l}\text { - Típicamente insensible a las condiciones } \\
\text { climáticas, cuando se opera en distancias } \\
\text { cortas; } \\
\text { - Medición directa de la velocidad; } \\
\text { - Operación en carriles múltiples utilizando } \\
\text { un solo instrumento; } \\
\text { - La instalación y el mantenimiento del } \\
\text { instrumento no implica el cierre de la vía. }\end{array}$ & $\begin{array}{l}\text { - No puede detectar vehículos detenidos; } \\
\text { - En las instalaciones desatendidas requiere que el } \\
\text { posicionamiento del sensor no sea cambiado; } \\
\text { - Instalación de alto costo. }\end{array}$ \\
\hline $\begin{array}{l}\text { Óptico } \\
\text { (Láser) }\end{array}$ & $\begin{array}{l}\text { - Transmite un haz de luz múltiple para la } \\
\text { medición precisa de la posición del vehículo, } \\
\text { la velocidad y clase; } \\
\text { - Operación en carriles múltiples utilizando } \\
\text { un solo instrumento; } \\
\text { - La instalación y el mantenimiento del } \\
\text { instrumento no implica el cierre de la vía. }\end{array}$ & $\begin{array}{l}\text { - La operación puede ser afectada por la presencia de } \\
\text { niebla o nieve; } \\
\text { - La instalación y el mantenimiento requiere la limpieza } \\
\text { de lentes de forma periódica; } \\
\text { - En las instalaciones desatendidas requiere que el } \\
\text { posicionamiento del sensor no sea cambiado; } \\
\text { - Instalación de alto costo. }\end{array}$ \\
\hline $\begin{array}{l}\text { Procesamiento } \\
\text { de imágenes }\end{array}$ & $\begin{array}{l}\text { - Monitorea múltiples carriles y múltiples } \\
\text { zonas de detección / carriles; } \\
\text { - Las zonas de detección son fáciles de añadir } \\
\text { y modificar; } \\
\text { - Proporciona una amplia detección y } \\
\text { monitoreo del tráfico. }\end{array}$ & $\begin{array}{l}\text { - La instalación y el mantenimiento requiere la limpieza } \\
\text { de lentes de forma periódica; } \\
\text { - El desempeño se ve afectado por el mal tiempo, como } \\
\text { niebla, lluvia o nieve; } \\
\text { - La operación confiable de la señal nocturna requiere } \\
\text { iluminación de la vía; } \\
\text { - Algunos modelos pueden afectarse por el movimiento } \\
\text { de la cámara causado por los vientos o la vibración de } \\
\text { la estructura de montaje. }\end{array}$ \\
\hline
\end{tabular}


Los equipos con sensores inductivos (objetivo principal de este artículo) se utilizan desde los años 60 en todo el mundo en diferentes aplicaciones de control de tráfico.

Debido a su bajo costo de instalación y mantenimiento y su alta confiabilidad, han sido considerados como los equipos más comunes para control electrónico de velocidad.

Inicialmente, estos instrumentos se limitan a notificar la presencia o ausencia de la masa metálica en el sensor inductivo. Sin embargo, con el advenimiento de las técnicas de procesamiento de señales digitales, nuevas características se han implementado a estos instrumentos (Nishimoto, 2006). Se destacan:

- Medición de la velocidad del vehículo;

- Detección de vehículos con poca masa metálica tales como motocicletas, bicicletas;

- Clasificación de los vehículos dentro de cada categoría, basándose en la información sobre el perfil magnético (Andreotti, 2001).

A pesar de sus evidentes ventajas del uso de la detección inductiva para control de tráfico, dicha tecnología tiene algunas debilidades, como se muestra en la Tabla 1.

Este artículo se centra en el problema del splash over. El fenómeno de splash over está presente en los carriles de tráfico adyacentes. La combinación de las propiedades físicas de los sensores inductivos con ciertas condiciones de configuración del equipo pueden causar su ocurrencia.

El splash over se produce debido a la extensión (spreading) de las líneas del campo magnético, formados alrededor de los devanados inductivos (Nishimoto, 2006). Cuando estas líneas extrapolan el carril, la detección correcta de la velocidad de los vehículos puede verse afectada de manera decisiva.

En este trabajo, se presenta en la Sección 2 el concepto de control de velocidad de tráfico a través del principio del circuito inductivo y la física que permite su funcionamiento, y por lo tanto, la descripción del proceso de la medición de la velocidad de los vehículos. En la Sección 3, se discute el fenómeno de splash over, presentando los resultados de las pruebas realizadas para esta investigación. Por último, en la Sección 4 presentamos las conclusiones.

\section{CONSIDERACIONES DEL CIRCUITO}

De manera rápida recordemos que los instrumentos que utilizan sensores inductivos pueden detectar los cambios de velocidad del vehículo en sus propiedades inductivas debido al paso de la estructura metálica del vehículo sobre su área de detección.

Estos instrumentos están formados básicamente por el bucle inductivo (inductive loop), cable de alimentación y una unidad de control electrónico.

El bucle inductivo está compuesto por una bobina que por lo general cuenta con tres a cinco vueltas de cable aislado enterrado en los surcos del pavimento. El cable de alimentación se utiliza para conectar la caja a la cabina. El hardware de la unidad de control electrónico es esencial para el funcionamiento del equipo. La unidad electrónica alimenta el sensor y se conecta típicamente con frecuencias de $10 \mathrm{kHz}$ a $200 \mathrm{kHz}$ (Magalhães, 2008).

Los equipos que utilizan sensores inductivos son capaces de detectar la presencia de una superficie metálica conductora mediante la alteración de las cantidades físicas del circuito RLC presente en este tipo de instrumentos.

La corriente que fluye en este circuito da lugar a un campo magnético alrededor de los alambres que forman bucles inductivos, según la ley de Ampère. Puesto que el circuito es alimentado por una corriente alterna tiene un flujo magnético que varía con el tiempo. Por lo tanto, cuando un vehículo cruza la zona de influencia del campo magnético generado por el bucle inductivo las corrientes de baja intensidad son inducidas (Fullerton, Mills y Kell, 1990).

Las corrientes inducidas generadas en las partes conductoras del vehículo dan lugar a otro campo magnético que interactúa con el campo establecido inicialmente en el bucle, provocando una inductancia mutua entre el bucle inductivo y el vehículo. Fullerton, Mills y Kell (1990) informan que el flujo 
magnético generado por la corriente en el vehículo en esta condición tiene dirección opuesta a la inducida por el flujo de corriente, como se espera según la ley de Lenz, causando una disminución en el campo magnético total inicialmente establecido alrededor del bucle.

El resultado directo de los cambios en el flujo magnético es la disminución de inductancia del bucle, puesto que la inductancia es proporcional al flujo magnético como se muestra a continuación (1).

$$
L=\frac{N \cdot \Phi_{B}}{i}
$$

Donde:

$L=$ inductancia;

$N$ = número de vueltas;

$\Phi B$ = flujo magnético;

$i=$ corriente eléctrica.

Sin embargo, las unidades electrónicas no detectan directamente el cambio en la inductancia. En general, poseen la capacidad de identificar el aumento de la frecuencia de resonancia del circuito o la disminución de la amplitud de la señal en el bucle como se muestra en Nishimoto (2006).

La frecuencia de resonancia de un circuito LCR impulsado por una corriente alterna (Klein, Gibson, 2006), tales como medidores de velocidad utilizados por los vehículos automotores, está dada por:

$$
f=\frac{1}{2 \pi \sqrt{L \cdot C}}
$$

Donde:

$\mathrm{f}=$ frecuencia del oscilador;

$\mathrm{L}=$ inductancia total del circuito;

$\mathrm{C}=$ capacitancia total del circuito.

Se puede demostrar usando la ecuación diferencial de los modelos de un circuito LCR con fuente externa presente:

$$
L \frac{d^{2} I}{d t^{2}}+R \frac{d I}{d t}+\frac{1}{C} I=\frac{d E}{d t}
$$

Donde:

$\mathrm{I}$ = corriente;

$E$ = fuerza electromotriz;

$\mathrm{R}=$ resistencia total del circuito.

La solución a la Ecuación (3) puede ser dada por (suponiendo que la fuente externa tiene una dependencia de tiempo dada, tal como se muestra a continuación):

$$
E(t)=E_{o} \exp (j w t) \quad I(t)=I_{o} \exp (j \alpha t)
$$

Al insertar las ecuaciones anteriores en (3), se puede obtener:

$$
\left[\alpha^{2} L+J R \alpha+\frac{1}{C}\right] I_{o} \exp (j \alpha t)=j \omega E_{o} \exp (j \omega t)
$$

Debido a que el sistema se ve obligado por la fuente, el circuito como un todo oscila con la frecuencia de la fuente, a saber:

$$
\alpha=\omega
$$

Resultando en:

$$
I_{o}=\frac{j \omega / L}{1 / L C^{-\omega^{2}+j \omega R} / L} E_{o}
$$

Al observar la Ecuación (5) vemos como la amplitud de la corriente alcanzará su valor máximo cuando:

$$
\omega^{2}=\frac{1}{L C}
$$

La frecuencia que permite alcanzar el valor máximo se conoce como la frecuencia natural de oscilación del circuito, y cuando la fuente externa es igual a esta frecuencia natural es normal decir que el circuito está en resonancia con la fuente externa.

Por lo tanto, la frecuencia de oscilación de un circuito resonante es dada, respectivamente, por las Fórmulas (7) y (8):

$$
w=\frac{1}{\sqrt{L C}} \mathrm{rad} / \mathrm{s}
$$




$$
f=\frac{1}{2 \pi \sqrt{L C}} H z
$$

Las Ecuaciones (7) y (8) muestran la dependencia de la frecuencia con la inductancia del circuito. Para mayor claridad en la relación, se puede derivar la frecuencia f para obtener $\mathrm{df} / \mathrm{dL}$ :

$$
\frac{d f}{d L}=\frac{1}{2 \pi} \frac{d(L C)^{-1 / 2}}{d L}
$$

A partir de (9) podemos entonces:

$$
\Delta f=-\frac{C}{4 \pi(L C)^{3 / 2}} \Delta L
$$

Dividiendo ambos lados de la ecuación, simplificando, llegamos a:

$$
\frac{\Delta f}{f}=-\frac{1}{2} \frac{\Delta L}{L}
$$

La Ecuación (11) muestra que la frecuencia normalizada varía con la mitad de la inductancia normalizada. El signo negativo significa que si existieran variaciones en la inductancia, debido a, por ejemplo, la presencia de la masa metálica de un vehículo que atraviesa, se corresponderá con un opuesto - un aumento de la frecuencia contraria. El aumento de la inductancia da lugar a una disminución de la frecuencia.

La detección, por la unidad electrónica, de la presencia de un vehículo con una velocidad por encima del valor permitido se produce cuando el cambio en la inductancia, debido a la interacción entre el bucle y el vehículo, excede un cierto umbral configurado previamente en el instrumento.

La unidad electrónica del equipo permite realizar diferentes ajustes de sensibilidad de detección. Esta sensibilidad define la cantidad de masa de metal conductora que debería estar presente en la región de influencia de los sensores en el vehículo detectado por el instrumento.

\subsection{Medición de velocidad con senso- res inductivos}

La medición de la velocidad de los vehículos es una de las principales aplicaciones de los sensores inductivos en el control del tráfico vehicular en Brasil. En este país, este sistema es ampliamente utilizado en los equipos medidores de velocidad, conjuntamente con los sensores dedicados que vigilan la violación de la luz roja en semáforos.

El sistema de control de velocidad utiliza generalmente dos o tres sensores inductivos que permanecen instalados en la calle o carretera monitoreada manteniendo una distancia fija.

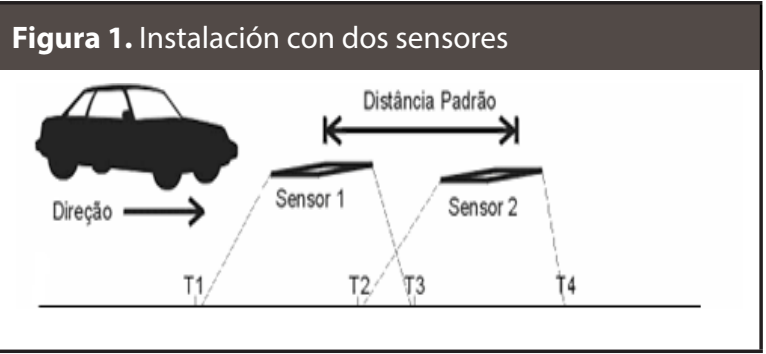

El funcionamiento de estos equipos se basa en la ecuación cinemática básica:

$$
V=\frac{d}{t}
$$

Donde:

$d=$ distancia entre los sensores;

$t=$ tiempo transcurrido entre el disparo del primer y segundo sensor.

La velocidad del vehículo se calcula a partir del tiempo transcurrido entre el primer y segundo sensor, que se produce cuando se alcanza el umbral de activación establecido en el equipo. Dado que la distancia entre los bucles es conocida, la velocidad se puede obtener usando la Ecuación (12).

\section{EL SPLASH OVER}

El splash over puede inducir a falsa detección (Coifman 2009). El fenómeno puede ser causado 
por un vehículo que viaja en un carril adyacente a aquel en donde está instalado el equipo de detección de velocidad.

Esta manifestación, no deseada, aparece cuando los enlaces operan a altos niveles de sensibilidad como consecuencia del control deseado por parte de la autoridad de tránsito para detectar vehículos pequeños, tales como motocicletas. Este problema también puede afectar a las instalaciones donde el sensor inductivo se coloca muy cerca de la división entre carriles de la misma vía.

Las líneas de campo magnético formadas alrededor de las bobinas del sensor no se restringen al perímetro del bucle. Por lo tanto dependen de la posición del bucle hacia la región del splash, el campo magnético puede invadir el carril adyacente, especialmente cuando se opera a altas sensibilidades. Con esta condición, incluso un pequeño cambio en las condiciones de equilibrio de circuito bucle / unidad electrónica, puede causar falsa identificación de un vehículo infractor.

La Figura 2 ilustra un ejemplo de ocurrencia de splash over. El bus interfiere con el bucle adyacente, lo que puede causar que el equipo detecte la presencia de un vehículo fantasma.

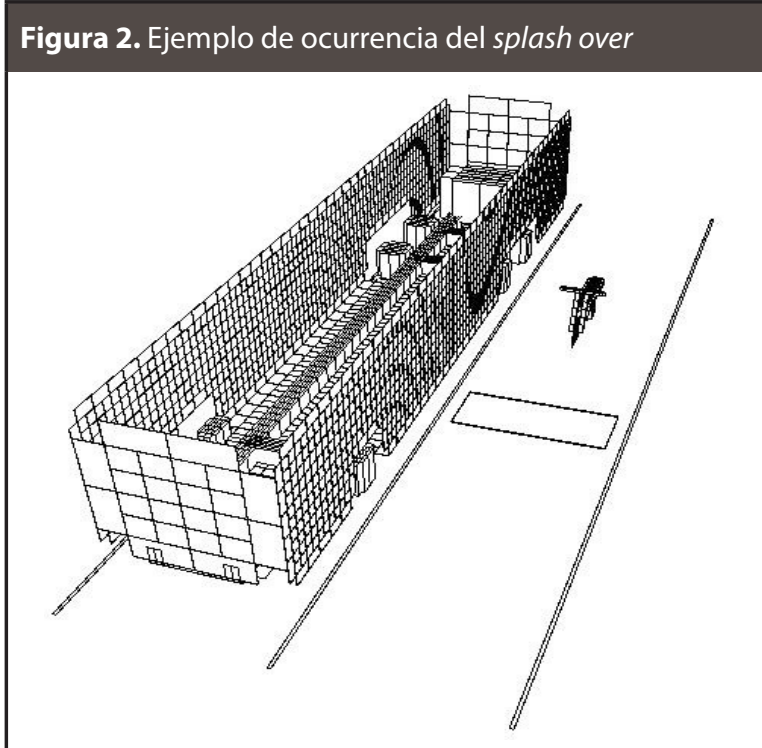

Según Andreoti (2001), los vehículos que generan más splash over son autobuses y carros. Ambos tienen pequeña altura por encima del suelo y gran masa metálica vertical (laterales del vehículo) que se mueven paralelamente cerca de las fronteras de los sensores.

\subsection{Prueba de splash over}

En esta investigación queremos certificar la ocurrencia real del splash over, y en cuáles condiciones puede estar presente este fenómeno no deseado.

Las pruebas se realizaron utilizando un equipo medidor de velocidad para vehículos a motor utilizado en varias ciudades de Brasil para el control de velocidad.

El medidor de velocidad está constituido básicamente por los dispositivos de detección y medición, procesamiento, almacenamiento y registro con las siguientes características:

- Dispositivos de detección y medición: consiste en 1 par de sensores inductivos y electrónicos Reno A\&E Modelo 222. Este módulo está dotado de componentes de alta relevancia metrológica.

- Procesamiento de dispositivos: consta de hardware y software capaz de procesar la información desde el dispositivo de detección y su medición. Este módulo es uno de los componentes necesarios para la operación del equipo.

- Dispositivo de almacenamiento: constituido de un disco duro capaz de almacenar los registros de las mediciones.

- Dispositivo de registro: cámara digital con capacidad de identificar el vehículo medido.

El instrumento fue instalado en un campo de prueba configurado para controlar la velocidad de un carril que utiliza un par de sensores inductivos.

Los sensores inductivos se fijaron en el pavimento siguiendo la instalación predeterminada del modelo, es decir, la distancia entre los sensores de 4 $\mathrm{m}$, teniendo ambos $2 \mathrm{~m}$ de largo y $0,80 \mathrm{~cm}$ de ancho (Figura 3). 
Figura 3. Dimensiones del sensor

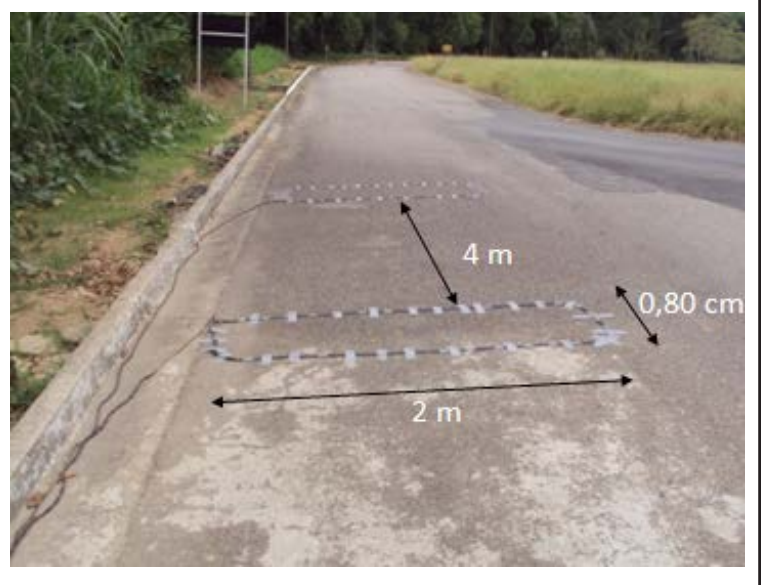

La unidad detectora de vehículos es responsable de la detección de la presencia de una masa de metal a través de los sensores inductivos conectados a ella. Esto es posible mediante la variación de magnitudes relacionadas con la inductancia del circuito, tales como la frecuencia o la amplitud. El equipo es capaz de informar los momentos en los que un vehículo entra y sale de la zona de medición, lo que permite medir el tiempo y la velocidad.

El modelo para este estudio utiliza la tabla de Reno A\&E para medir cambios en la frecuencia resonante de cada uno de los circuitos formados por los sensores, cables de conexión y unidad de detección. El objetivo es determinar si un vehículo ha entrado en la zona de detección. El detector aplica un voltaje de control al circuito de generación. El paso de un conductor metálico hace que la frecuencia de resonancia del circuito aumente. Si este cambio excede de un umbral seleccionable, determinado por el ajuste de sensibilidad, el detector activa una señal de salida (Reno 2006).

Entre otros ajustes en la placa, es posible ajustar la sensibilidad de detección del instrumento. El modelo Reno A\&E permite ocho diferentes niveles de umbrales de detección. Por lo tanto, dependiendo de la configuración, se puede descartar la captura de pequeños vehículos como bicicletas y motocicletas.
El manual de la placa de detección utilizado en la prueba indica cómo la inductancia del circuito debe variar en cada nivel de sensibilidad para que se produzca la detección.

La metodología consistió en realizar la prueba para vehículos con dimensiones más allá de los sensores con el fin de verificar la capacidad de detección y medición de la velocidad del equipo.

Dos parámetros se variaron durante las pruebas: la sensibilidad de detección del equipo y la distancia a los sensores del sobrepaso del vehículo de prueba.

La prueba utilizada para parámetros iniciales en la placa de detección corresponde al valor AL/L $=0,32 \%$ a $20 \mathrm{~cm}$ de distancia del borde del sensor.

TABLA 1. NIVELES DE SENSIBILIDAD Y UMBRALES DE DETECCIÓN (RENO, 2006)

\begin{tabular}{c|c}
$\begin{array}{c}\text { Niveles de } \\
\text { sensibilidad }\end{array}$ & $\begin{array}{c}\text { Niveles de la sensibilidad de } \\
\text { detección }(\Delta \mathrm{L} / \mathrm{L} \%)\end{array}$ \\
\hline 0 & 1,28 \\
\hline 1 & 0,64 \\
\hline 2 & 0,32 \\
\hline 3 & 0,16 \\
\hline 4 & 0,08 \\
\hline 5 & 0,04 \\
\hline 6 & 0,02 \\
\hline 7 & 0,01 \\
\hline
\end{tabular}

Figura 4. Distancias utilizadas en la prueba de splash over

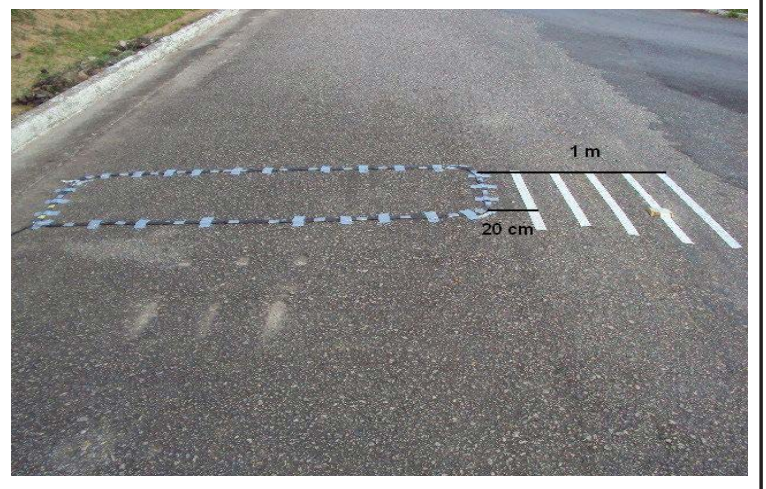

Revista EIA Rev.EIA.Esc.Ing.Antioq / Universidad EIA 
TABLA 2. RESULTADOS DE LA SIMULACIÓN DEL SPLASH OVER CON $\triangle \mathrm{L} / \mathrm{L} \%=0,32 \%$

\begin{tabular}{c|c|c}
\multirow{2}{*}{} & \multicolumn{2}{|c}{ Distancia de sobrepaso del vehículo a los sensores laterales (d) } \\
\cline { 2 - 3 } & Medición & Record \\
\cline { 2 - 3 } & No & No \\
\hline 1 & No & No \\
\hline 2 & No & No \\
\hline 3 & No & No \\
\hline 4 & No & No \\
\hline 5 & No & No \\
\hline 6 & No & No \\
\hline 7 & No & No \\
\hline 8 & No & No \\
\hline 9 & No & No \\
\hline 10 & &
\end{tabular}

TABLA 3. RESULTADOS DE LA SIMULACIÓN DE SPLASH OVER CON $\triangle \mathrm{L} / \mathrm{L} \%=0,04 \%$

\begin{tabular}{|c|c|c|c|c|c|c|}
\hline & \multicolumn{6}{|c|}{ Distancia de sobrepaso del vehículo a los sensores laterales (d) } \\
\hline & \multicolumn{2}{|c|}{$\mathrm{d} \geq 20 \mathrm{~cm}$} & \multicolumn{2}{|c|}{$40 \mathrm{~cm} \leq \mathrm{d}<60 \mathrm{~cm}$} & \multicolumn{2}{|c|}{$60 \mathrm{~cm} \leq \mathrm{d}<80 \mathrm{~cm}$} \\
\hline & $\begin{array}{l}\text { Medición } \\
\text { Realizada }\end{array}$ & $\begin{array}{l}\text { Informe } \\
\text { fotográfico } \\
\text { generado }\end{array}$ & $\begin{array}{l}\text { Medición } \\
\text { Realizada }\end{array}$ & $\begin{array}{l}\text { Informe } \\
\text { fotográfico } \\
\text { generado }\end{array}$ & $\begin{array}{l}\text { Medición } \\
\text { Realizada }\end{array}$ & $\begin{array}{l}\text { Informe } \\
\text { fotográfico } \\
\text { generado }\end{array}$ \\
\hline 1 & Sí & Sí & Sí & Sí & No & No \\
\hline 2 & Sí & Sí & Sí & Sí & No & No \\
\hline 3 & Sí & Sí & Sí & Sí & No & No \\
\hline 4 & Sí & Sí & Sí & Sí & No & No \\
\hline 5 & Sí & Sí & Sí & Sí & No & No \\
\hline 6 & Sí & Sí & Sí & Sí & No & No \\
\hline 7 & Sí & Sí & Sí & Sí & No & No \\
\hline 8 & Sí & Sí & Sí & Sí & No & No \\
\hline 9 & Sí & Sí & Sí & Sí & No & No \\
\hline 10 & Sí & Sí & Sí & Sí & No & No \\
\hline
\end{tabular}

Realizamos diez (10) sobrepasos con el vehículo de prueba en cada conjunto de intervalos y observamos el comportamiento del equipo para cada situación. La velocidad de medición se aumentó a un intervalo de $20 \mathrm{~cm}$, determinando así la nueva distancia a la que el vehículo debería pasar cada prueba. Sin embargo, cuando el equipo ya no fue ca- paz de medir la velocidad, se estableció una nueva sensibilidad y se realizaron varios sobrepasos con el vehículo. El procedimiento se repitió hasta llegar a la distancia en la que el equipo ya no fue capaz de medir la velocidad, es decir en el último nivel de configuración de sensibilidad disponible en el detector modelo utilizado para la prueba. 


\begin{tabular}{|c|c|c|c|c|c|c|}
\hline & \multicolumn{6}{|c|}{ Distancia de sobrepaso del vehículo a los sensores laterales (d) } \\
\hline & \multicolumn{2}{|c|}{$60 \mathrm{~cm} \leq \mathrm{d}<80 \mathrm{~cm}$} & \multicolumn{2}{|c|}{$80 \mathrm{~cm} \leq \mathrm{d}<100 \mathrm{~cm}$} & \multicolumn{2}{|c|}{$100 \mathrm{~cm} \leq \mathrm{d}<120 \mathrm{~cm}$} \\
\hline & $\begin{array}{l}\text { Medición } \\
\text { Realizada }\end{array}$ & $\begin{array}{l}\text { Informe } \\
\text { fotográfico } \\
\text { generado }\end{array}$ & $\begin{array}{l}\text { Medición } \\
\text { Realizada }\end{array}$ & $\begin{array}{l}\text { Informe } \\
\text { fotográfico } \\
\text { generado }\end{array}$ & $\begin{array}{l}\text { Medición } \\
\text { Realizada }\end{array}$ & $\begin{array}{l}\text { Informe } \\
\text { fotográfico } \\
\text { generado }\end{array}$ \\
\hline 1 & Sí & Sí & Sí & Sí & No & No \\
\hline 2 & Sí & Sí & Sí & Sí & No & No \\
\hline 3 & Sí & Sí & Sí & Sí & No & No \\
\hline 4 & Sí & Sí & Sí & Sí & No & No \\
\hline 5 & Sí & Sí & Sí & Sí & No & No \\
\hline 6 & Sí & Sí & Sí & Sí & No & No \\
\hline 7 & Sí & Sí & Sí & Sí & No & No \\
\hline 8 & Sí & Sí & Sí & Sí & No & No \\
\hline 9 & Sí & Sí & Sí & Sí & No & No \\
\hline 10 & Sí & Sí & Sí & Sí & No & No \\
\hline
\end{tabular}

\section{TABLA 5. RESULTADOS DE LA SIMULACIÓN DE SPLASH OVER CON $\triangle \mathrm{L} / \mathrm{L} \%=0,01 \%$}

Distancia de sobrepaso del vehículo a los sensores laterales (d)

\begin{tabular}{|c|c|c|c|c|}
\hline & \multicolumn{4}{|c|}{ Distancia de sobrepaso del vehículo a los sensores laterales (d) } \\
\hline & \multicolumn{2}{|c|}{$100 \mathrm{~cm} \leq \mathrm{d}<120 \mathrm{~cm}$} & \multicolumn{2}{|c|}{$\mathrm{d} \geq 120 \mathrm{~cm}$} \\
\hline & Medición & $\begin{array}{l}\text { Informe fotográfico } \\
\text { generado }\end{array}$ & Medición & $\begin{array}{l}\text { Informe fotográfico } \\
\text { generado }\end{array}$ \\
\hline 1 & Sí & Sí & No & No \\
\hline 2 & Sí & Sí & No & No \\
\hline 3 & Sí & Sí & No & No \\
\hline 4 & Sí & Sí & No & No \\
\hline 5 & Sí & Sí & No & No \\
\hline 6 & Sí & Sí & No & No \\
\hline 7 & Sí & Sí & No & No \\
\hline 8 & Sí & Sí & No & No \\
\hline 9 & Sí & Sí & No & No \\
\hline 10 & Sí & Sí & No & No \\
\hline
\end{tabular}

En las pruebas de simulación del splash over se utilizaron cuatro (4) niveles de sensibilidad y se registraron cinco (5) diferentes intervalos en los sensores inductivos.
El equipo operando con la sensibilidad inicial no detectó el sobrepaso del vehículo a $20 \mathrm{~cm}$ de distancia de los sensores, como se muestra en la Tabla 2. Por lo tanto, de acuerdo con la metodología establecida, se 
realizó un nuevo ajuste para hacer que el equipo fuera más sensible, lo que permitió la medición de la velocidad en la región exterior de los sensores.

El segundo nivel de sensibilidad utilizado en la prueba fue de 0,04\% (Tabla 3). En esta configuración con el vehículo pasando a una distancia de 20 $\mathrm{cm}$ del sensor, el instrumento fue capaz de medir la velocidad del vehículo de prueba y emitir el registro fotográfico correspondiente. Con esta sensibilidad el equipo también detectó la masa metálica del vehículo en todos los sobrepasos realizados a $40 \mathrm{~cm}$ de distancia del borde del sensor.

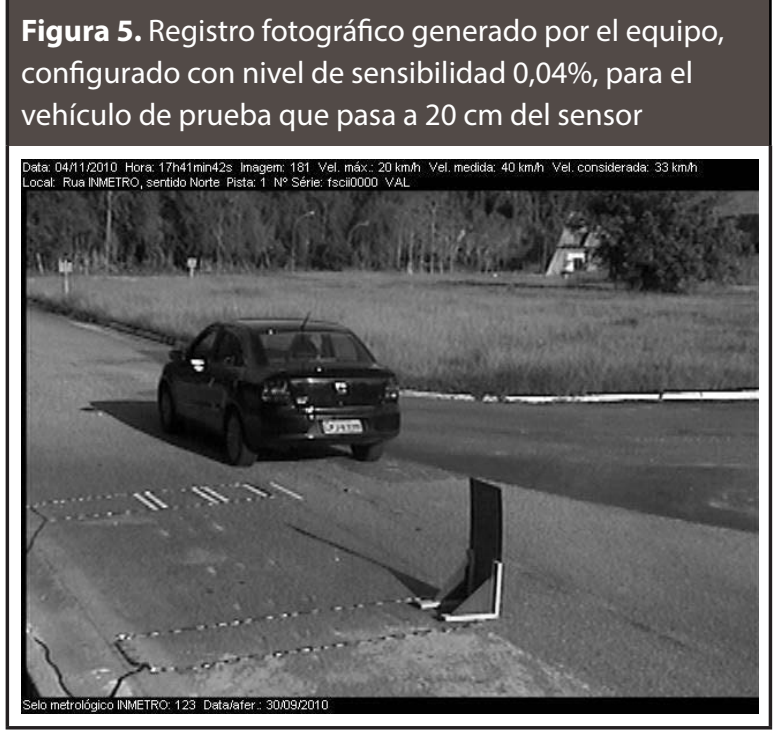

Figura 6. Registro fotográfico generado por el equipo, configurado con nivel de sensibilidad de $0,02 \%$, para el vehículo de prueba atravesando a $80 \mathrm{~cm}$ del sensor

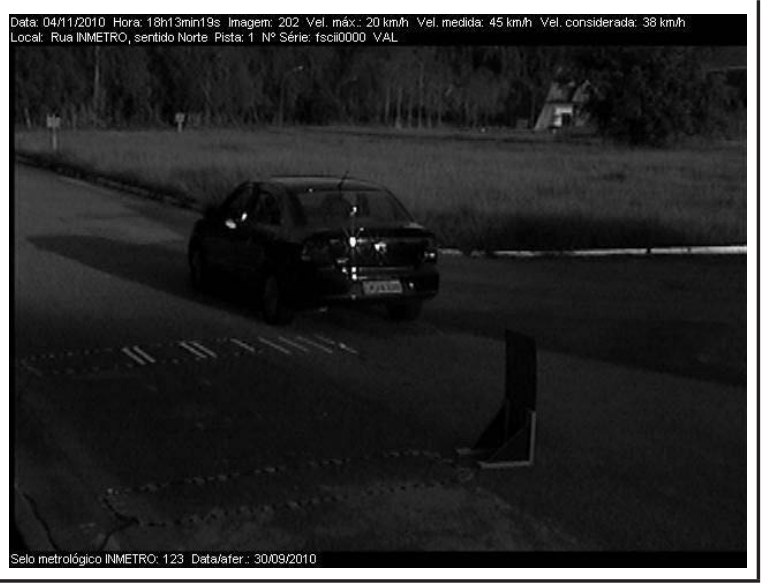

El tercer nivel de sensibilidad utilizado fue 0,02\%. En esta etapa del proceso, inicialmente, el vehículo de prueba pasó a $60 \mathrm{~cm}$ del borde del bucle. El equipo midió la velocidad y la fotografía se emitió a una distancia de $80 \mathrm{~cm}$ desde el borde del bucle, como se muestra en la Tabla 4.

El cuarto y último nivel de sensibilidad fue del 0,01\% (Tabla 5). Con el fin de encontrar la distancia máxima desde el borde del bucle, donde la masa metálica es capaz de interferir significativamente con el campo magnético generado alrededor de los sensores. El instrumento se ajustó para funcionar en la mayor sensibilidad posible. Con esta configuración, el equipo mide la velocidad (Figura 7) con el vehículo de prueba a través de una distancia de al menos $1 \mathrm{~m}$ desde el borde del sensor.

Figura 7. Registro fotográfico generado por el equipo,
configurado con nivel de sensibilidad de 0,01\%, para el
vehículo de prueba que atraviesa a $100 \mathrm{~cm}$ del sensor

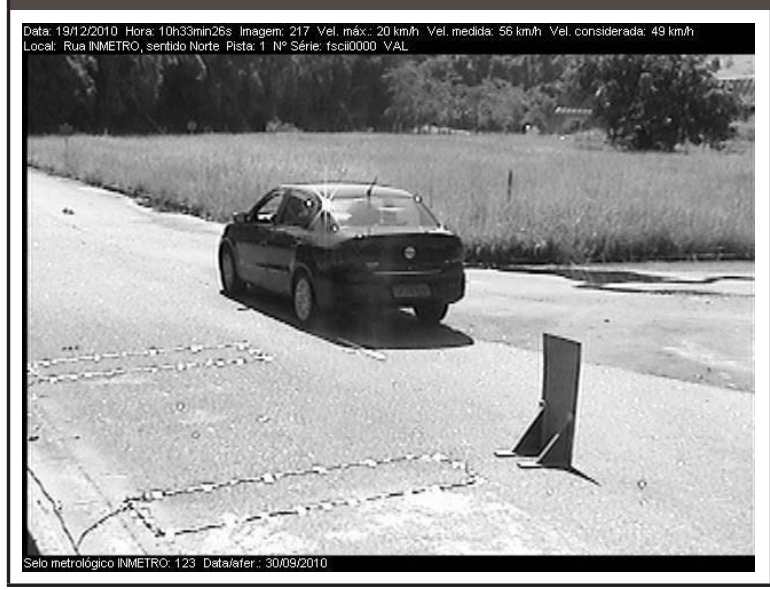

Como era de esperarse, la distancia máxima desde el borde del bucle en el que la masa metálica fue capaz de interferir significativamente con el campo magnético creado alrededor de los sensores se produjo con el equipo regulado para operar al nivel más alto de sensibilidad posible. Con este ajuste, el equipo detectó y midió la velocidad (Figura 4) del vehículo de prueba a 1 metro del borde del cable sensor (Monteiro, 2012). 
Este resultado es razonable, ya que se considera que el vehículo al aumentar la distancia del sensor implica una menor cantidad de masa de metal conductora en la interacción con el campo magnético local. Adicionalmente, el campo magnético en la región externa al sensor se enfrenta a una disminución gradual cerca de los sensores existentes. La consecuencia de la asociación de estas dos condiciones es que la presencia de un vehículo en regiones fuera del sensor causará una pequeña variación en la inductancia del circuito, parecida a la condición producida con el paso de un vehículo sobre los sensores. Por lo tanto, los ajustes a las sensibilidades son necesarios con el fin poder detectar los cambios más pequeños.

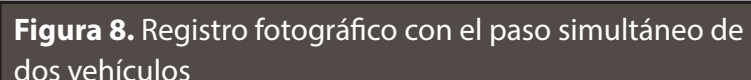
dos vehículos

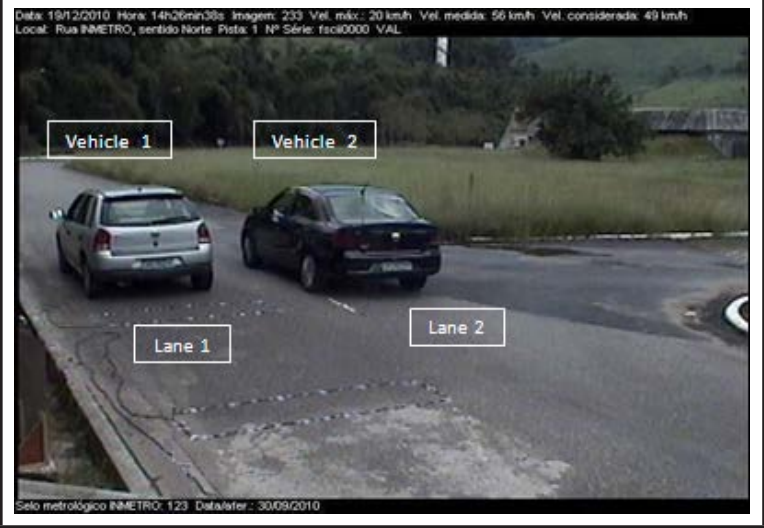

Figura 9. Registro fotográfico generado por el equipo con la configuración normal de cámara utilizada para el registro de la infracción

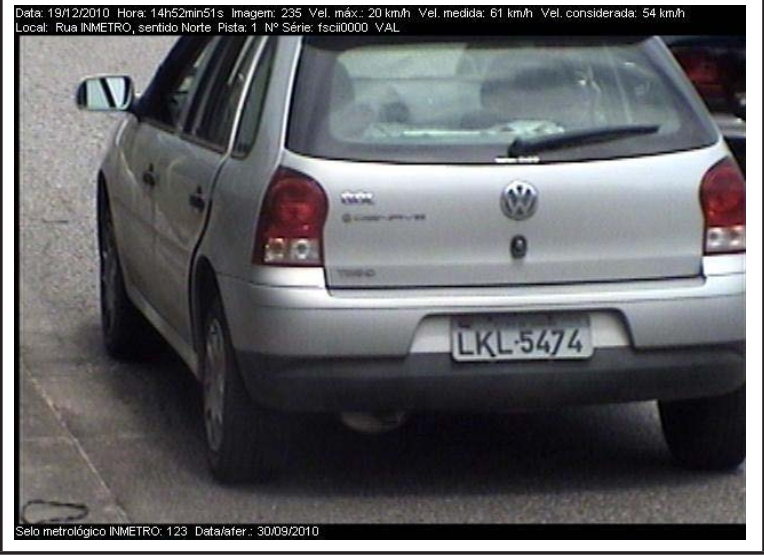

Con el objetivo de mostrar cómo puede ocurrir la falsa detección debido al splash over fueron simuladas situaciones especialmente críticas con la ayuda de dos vehículos de prueba.

En estas simulaciones el equipo se ajustó para funcionar con el nivel de sensibilidad de 0,04\%, para poder captar un vehículo que atraviesa hasta $40 \mathrm{~cm}$ del borde del bucle, como se muestra en la Tabla 03 Tabla 3.

La Figura 5 muestra un vehículo en el carril 1, donde está instalado el par de sensores, y el otro vehículo en el carril 2. El vehículo 1 tiene una velocidad menor que la velocidad máxima establecida en el equipo para realizar la detección, por otra parte, el segundo vehículo en el carril 2 tiene una velocidad superior a la velocidad límite en la cual el equipo puede detectar la violación.

El vehículo 1 de acuerdo con el splash over podría ser identificado como el responsable de la velocidad medida por el equipo, cuando en realidad el exceso velocidad fue responsabilidad del vehículo 2 .

El registro fotográfico entonces, como se muestra en la Figura 6, hace que sea más factible la falsa identificación del responsable de la velocidad. El ajuste del zoom de la cámara no muestra la imagen del otro vehículo en el carril adyacente, responsable del splash over.

\section{CONCLUSIONES}

El splash over es un fenómeno inherente al utilizar sensores inductivos para el control de exceso de velocidad. Como se describió en este trabajo, la presencia de vehículos que circulan en carriles adyacentes puede causar falsas detecciones o errores en la medición de la velocidad.

La simulación mostró que el equipo, cuando se ajusta para operar a altos niveles de sensibilidad, es capaz de detectar un vehículo que viaja fuera del perímetro de los sensores. Tales ajustes extremos pueden ser requeridos para la detección de vehículos pequeños o de gran distancia del suelo. 
El problema del splash over se hace más crítico ya que la zona de detección y la intensidad del campo magnético formado alrededor de los sensores dependen de las condiciones particulares de la instalación de cada instrumento. No es posible establecer un ajuste de sensibilidad estándar que cumpla con todos los prototipos y modelos.

El ajuste de sensibilidad realizado en el tablero de detector puede ser considerado como el factor más relevante en la configuración del equipo. Un valor menos sensible impediría la detección de la velocidad de los vehículos que viajan en el carril adyacente de medición, pero con este ajuste, el instrumento sería incapaz de capturar el paso de vehículos como motocicletas que poseen menor masa metálica y son distantes del suelo. Por el contrario, ajustes muy sensibles garantizan la medición de todo tipo de vehículos, pero permiten que el equipo registre falsos valores de velocidad.

Encontrar el equilibrio que permita el funcionamiento satisfactorio del medidor de velocidad no siempre será fácil. La calibración del instrumento debe realizarse durante la instalación y por lo general se lleva a cabo de forma empírica.

Por lo tanto, después de la instalación o mantenimiento, el medidor de velocidad puede operar, aunque involuntariamente, con el fenómeno de splash over.

Existen maneras de minimizar el efecto no deseado del splash over.

La instalación de sensores de respuesta retardada (lagged sensors), la no instalación de sensores cerca de las divisiones de los carriles y de la zona de medición gráfica de bandas adyacentes, que es la región de influencia del campo magnético generado por el instrumento de registro fotográfico, son medidas que si se utilizan podría proporcionar soluciones para esta anomalía.

Por último, es importante destacar que esta investigación se utilizó para el ajuste de las regulaciones técnicas de metrología para la velocidad en los vehículos automotores en Brasil.

\section{REFERENCIAS}

Andreoti, Marcel. Inductive sensing for automotive vehicles. Curitiba: Technological Center Federal do Paraná, 2001.52 p.

Coifman, Benjamin. Lenght classfication vehicle based on freeways form single loop detectors. Columbus: Nextrans, 2009. 13-14 p.

Fullerton, Iris; Kell, James and Mills, Milton. Traffic Detector Handbook - Second Edition, Virginia: Federal Higway Administration, 1990. 18-19 p.

Klein, Lawrence; Gibson, David and Mills, Milton. Traffic Detector Handbook - Third Edition. Virginia: Federal Higway Administration, 2006. 81-84 p.

Magalhães, Hermes. Analysis of high-resolution magnetic profiles of the inductive loop sensors for classification of vehicles. Belo Horizonte: Federal University of Minas Gerais, 2008. 44-46 p.

Monteiro, Silvio. Study on the inclusion of the measurement zone in legal control of speed meters of automotive vehicles. Rio de Janeiro: National Institute of Metrology, Quality and Technology, 2012. 55-75 p.

Nishimoto, Régis. New geometries inductive loop. Curitiba: Technological University of Paraná, 2006. 4-6 p.

Reno A\&E. Operation manual reno A\&E - model 222. Reno: Reno ae, 2006. 13-16 p.

Rizzotto, Rodolfo. Accidents do not happen. Rio de Janeiro: National School of Insurance, 2010. 56 p.

World Health Organization. Road traffic injuries. 2004: [Accessed on May 2012] Available at: < www.who. int/features/2004/road_safety $>$.

\section{PARA CITAR ESTE ARTÍCULO / TO REFERENCE THIS ARTICLE / PARA CITAR ESTE ARTIGO /}

Monteiro, S.; Réche, M.; de Assis, A.S. (2017). Presencia no deseada del splash over en equipos que utilizan sensores inductivos para el monitoreo de la velocidad controlada en vehículos automotores. Revista EIA, 14(27), enero-junio, pp. 97-109. [Online]. Disponible en: https://doi.org/10.24050/ reia.v14i27.1160 